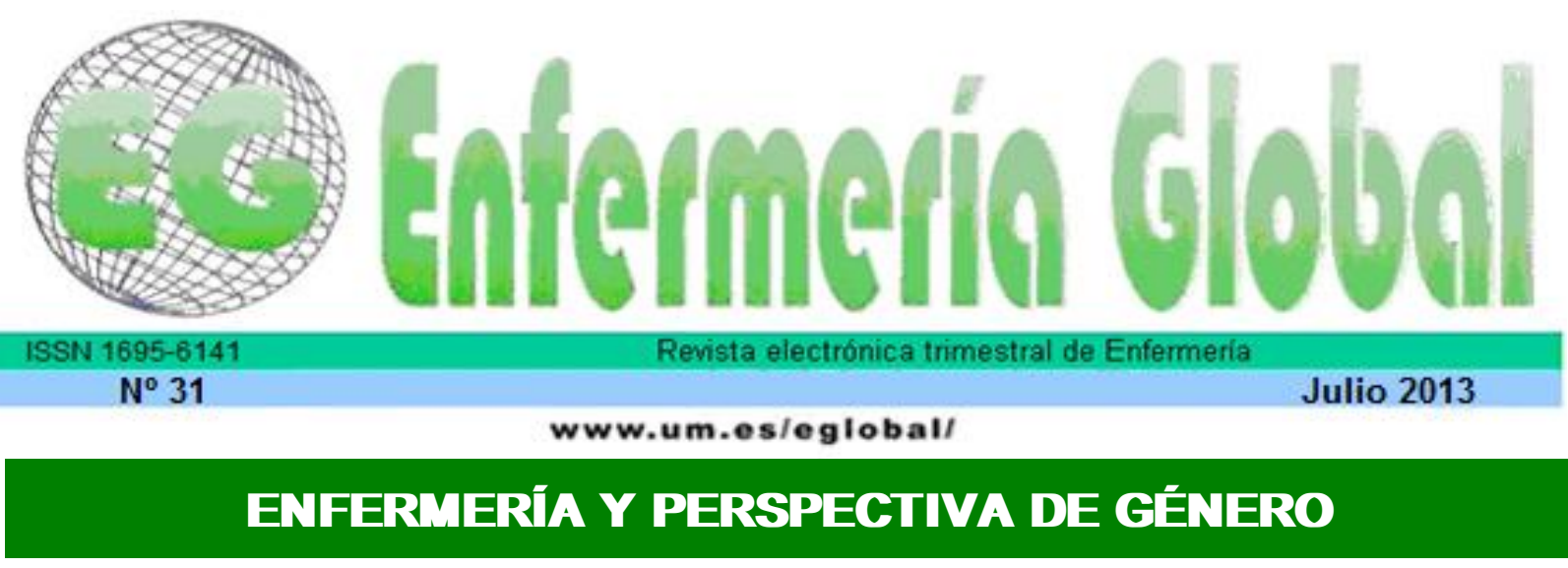

\title{
Lactancia materna: prevención de problemas tempranos en las mamas mediante una técnica de amamantamiento eficaz
}

Breastfeeding problems prevention in early breast feeding through effective technique

\author{
*Fernández Medina, Isabel María **González Fernández, Carmen Tamara \\ *Diplomada en Enfermería y Fisioterapia. Servicio Andaluz de Salud. Hospital Torrecárdenas \\ (Almería). E-mail: isabelmariafm@hotmail.com **Enfermera. Licenciada en Antropología Social y \\ Cultural. Servicio Andaluz de Salud. Hospital Virgen de las Nieves (Granada).
}

Palabras clave: lactancia materna; grietas; mastitis; ingurgitación; prevención

Keywords: Breastfeeding; fissures; mastitis; engorgement; prevention

\section{RESUMEN}

Introducción: Actualmente la mayoría de las madres conocen la importancia de la lactancia materna tanto para la madre como para el hijo desde el punto de vista nutritivo y de desarrollo así como afectivo y de prevención de patologías; pero algunas madres desconocen que una técnica de lactancia inadecuada puede llegar a ocasionar problemas tempranos en las mamas y con ello, en numerosas ocasiones, el abandono temprano del amamantamiento.

Objetivos: En este estudio se plantean las principales alteraciones mamarias puerperales que pueden estar asociadas a una técnica de lactancia ineficaz.

Metodología: Se ha realizado una búsqueda bibliográfica en las siguientes bases de datos: Medline, Pubmed y The Cochrane Database of Systematic Reviews. Se incluyen los trabajos relacionados con el objetivo del presente texto.

Resultados: Los estudios consultados evalúan la asociación entre la aparición de problemas mamarios y la utilización de una técnica de lactancia inadecuada y la eficacia de los tratamientos tras la aparición de las lesiones.

Conclusiones: La asociación entre la aparición de problemas tempranos en las mamas asociados a una técnica de lactancia materna inadecuada es evidente. Se concluye que la prevención es el mejor tratamiento, lugar donde desarrolla una importante labor el profesional sanitario.

\section{ABSTRACT}

Introduction: Currently, most mothers know the importance of breastfeeding for both the mother and the child from the nutritional point of view and emotional development as well as prevention and pathologies, but some mothers know that inadequate breastfeeding technique 
can cause early problems in the breasts and thus, in many cases, the early abandonment of lactation.

Objective: In this study the main puerperal breast abnormalities that may be associated with ineffective breastfeeding technique, due to insufficient maternal education about it are set out.

Method: We performed a literature research in the following databases: Medline, Pubmed and the Cochrane Database of Systematic Reviews. Works related to the objective of this text are included.

Results: Consulted studies evaluated the association between the onset of breast problems and the use of inappropriate lactation technique and the efficacy of treatments after the onset of the lesions.

Conclusions: The association between the occurrences of early problems in the breast associated with breastfeeding technique is evident. We conclude that prevention is the best treatment, where important work develops a healthcare professional.

\section{INTRODUCCIÓN}

Según la OMS, la leche materna constituye indiscutiblemente el mejor alimento que la madre puede ofrecer al recién nacido durante los primeros seis meses de vida. Posteriormente, hasta los dos años o más, los lactantes pueden seguir con la lactancia materna complementada con otros alimentos nutritivos ${ }^{(1)}$.

La leche materna aporta todos los nutrientes que el niño necesita en sus primeros meses de vida y sigue cubriendo la mitad o más de las necesidades nutricionales del niño durante el segundo semestre de vida y hasta un tercio durante el segundo año ${ }^{(2)}$.

Sin embargo aunque la capacidad de lactar es innata e instintiva en los mamíferos, en la mujer esta capacidad debe ser desarrollada y apoyada mediante una educación adecuada para lograr una lactancia exitosa y placentera ${ }^{(1,2)}$.

\section{Técnica de amamantamiento}

El periodo de gestación constituye el mejor momento para informar a las madres sobre la lactancia materna y como llevarla a cabo con éxito, si deciden amamantar.

Aunque la lactancia materna se considera un proceso natural, tanto la madre como el bebé precisan de un óptimo aprendizaje ya que el bebé ha de aprender a succionar correctamente el pecho y la madre ha de aprender las pautas de un posicionamiento correcto $^{(3)}$.

La primera toma ha de iniciarse lo más precoz posible, en la sala de partos, favorecida por el contacto piel con piel y aprovechando el primer periodo de reactividad del recién nacido. El contacto piel con piel favorece la adaptación del bebé a la vida extrauterina y favorece el vínculo con la madre y la lactancia. Una vez conseguida una primera toma correcta, se favorece el éxito de las siguientes tomas ${ }^{(1)}$.

La posición del lactante colocado al pecho es fundamental, debe tomar el pecho de frente, a la altura del pezón, su cuerpo debe estar en íntimo contacto con el de su 
madre "vientre contra vientre", su nariz debe estar a la misma altura que el pezón. El labio superior e inferior deben estar abiertos y evertidos para acoplarse a la areola. El niño nunca debe chupar solo el pezón.

Hay que evitar que el niño este demasiado alto y que tenga que flexionar el cuello y su nariz quede aplastada contra el pecho.

La madre debe situarse en una posición cómoda, si el pecho es demasiado grande se puede sujetar desde la base o en forma de copa, pero ha de evitarse que los dedos de la madre dificulten la extracción de leche o impidan que el bebé introduzca el pecho en su boca.

Los signos que indican una succión adecuada son: la mandíbula se mueve rítmicamente acompañada de las sienes y las orejas, la boca se encuentra bien abierta y los labios evertidos, nariz, mejillas y mentón tocan el pecho, realiza pausas de succión-deglución ocasionales, deglución audible y satisfacción después de la toma. Si las mejillas se succionan hacia dentro, el niño no estará lactando adecuadamente $^{(1,2)}$.

No se debe retirar al niño bruscamente del pecho ya que se puede dañar el pezón. Hay que romper antes el vacio de succión, introduciendo entre las comisuras de la boca un dedo.

La madre puede adoptar cualquier posición, tanto tumbada como sentada, solo se ha de tener en cuenta que el niño quede con su boca de frente a la altura del pecho, sin que tenga que girar, flexionar o extender el cuello e introduzca tanto el pezón como la areola en su boca. La posición también debe ser confortable para la madre.

Son importantes las tomas frecuentes aunque el ritmo de cada bebé es diferente, es aconsejable identificar los signos que indican que el bebé está dispuesto a mamar (como sacar la lengua, chuparse los puños, removerse en la cuna, etc.), ya que será más fácil conseguir una toma efectiva al ser el llanto un signo tardío de hambre que le dificulta al recién nacido el buen agarre al pecho. Por ello, las tomas al pecho deben ser a demanda. El principal estímulo que induce la producción de leche es la succión del niño, por lo tanto, cuantas más veces toma el pecho de la madre, más leche se produce $^{(3)}$.

La duración de las tomas es variable, pero es aconsejable el vaciado completo de un pecho antes de ofrecer el otro, debido a que la leche del final de la mamada es la más rica en grasa y la que provoca la sensación de saciedad y hace que el lactante deje de mamar cuando ya ha recibido la leche suficiente.

Si el niño no vacía completamente el último pecho, la toma siguiente deberá iniciarse en este pecho ${ }^{(1,3)}$.

El uso del chupete es un tema controvertido debido a que calma al bebé y tiene una función importantísima en la prevención del síndrome de muerte súbita del lactante. Hoy en día se aconseja su uso a partir del mes de nacimiento cuando la técnica de lactancia está debidamente instaurada, ya que su uso a edades más tempranas puede interferir en el éxito de la lactancia materna ${ }^{(4)}$.

Los principales signos de amamantamiento ineficaz son: 
- Pérdida de peso del lactante mayor del $7 \%$ respecto al peso del nacimiento.

- Pérdida continuada de peso después del tercer día.

- Menos de 3 deposiciones en 24 horas en los primeros días.

- Heces meconiales después del cuarto día.

- Lactante irritable e inquieto o somnoliento o que rehusa las tomas.

- Deglución no audible durante las tomas.

- No hay cambio discernible en el peso o tamaño de los pechos y no hay cambio aparente en la composición y el volumen de la leche entre el tercer y quinto día.

- Dolor de pezones persistente o creciente.

- Congestión mamaria que no mejora amamantando.

- Lactante que no ha empezado a ganar peso después del quinto día.

- Lactante que no ha recuperado el peso del nacimiento a las dos semanas ${ }^{(2)}$.

\section{MATERIAL Y MÉTODO}

Se ha realizado una búsqueda sistemática en las siguientes bases de datos: Medline, Pubmed y The Cochrane Database of Systematic Reviews. La búsqueda se restringió a los idiomas inglés y español, utilizando como descriptores los siguientes términos: breast-feeding, mastitis, fisures, engorgement y prevention.

Han sido incluidos trabajos publicados entre 1989 y 2011.

Los artículos utilizados han sido estudios clínicos y de revisión bibliográfica sobre la prevalencia en lactancia materna asociada a educación maternal, las técnicas más adecuadas de amamantamiento y la prevención de problemas asociados a la lactancia.

\section{RESULTADOS}

\section{Patología mamaría asociada a la lactancia materna}

Tras el inicio de la lactancia materna en el ámbito hospitalario, la continuidad y él éxito de la misma depende muchas veces de las dificultades que surgen en los primeros días como dolor, depresión post-parto, congestión mamaria o dudas respecto a la técnica de lactancia ${ }^{(1)}$.

La intervención y el seguimiento de los profesionales de la salud en los primeros días de inicio de la lactancia materna según los estudios consultados se ha considerado como un factor positivo en el éxito de la lactancia materna así como un indicador relevante en la disminución de las tasas de abandono precoz de la lactancia ${ }^{(5)}$. Renfrew destacó la importancia de educar y preparar a los profesionales de la salud para promover y apoyar la lactancia materna, como herramienta de prevención de patologías mamarias ${ }^{(6)}$.

Los estudios más recientes afirman que otro de los factores más influyente para el mantenimiento y éxito de la lactancia materna es la educación materna iniciada desde el periodo prenatal ${ }^{(1)}$. 
Las principales alteraciones mamarias son grietas en el pezón, congestión mamaria y mastitis. La incidencia de estas patologías puede variar entre el $34 \%$ y el $96 \%$ y hasta un tercio de las madres que experimentan este tipo de complicaciones abandona la lactancia materna ${ }^{(4)}$.

- Dolor y grietas en el pezón

El dolor en los pezones es frecuente que se produzca al inicio de la lactancia materna y normalmente se debe a la presión negativa producida en los conductos galactóforos que todavía no están llenos de leche. Normalmente al instaurarse definitivamente la lactancia el dolor suele desaparecer y si perdura se asocia con un mal acoplamiento del niño al lactar ${ }^{(1,2)}$.

Las grietas en el pezón suelen aparecer en los primeros días de iniciada la lactancia, cuando la posición al pecho y el acoplamiento boca-pezón no es el correcto. Estas constituyen uno de los principales motivos de abandono de la lactancia. El mal agarre del recién nacido origina las grietas al agredir el pezón con sus encías o al comprimirlo con la lengua contra el paladar duro ${ }^{(7,8)}$.

Ante cualquier dolor al amamantar o grietas en el pezón, siempre se debe observar la mamada, observar el pezón después de la mamada y examinar la succión del niño.

Existe una correlación positiva entre la tensión mamaria en la subida de la leche y las lesiones en el pezón, que podrían producirse porque en estos casos es más difícil para el lactante agarrarse al pecho.

En un estudio de corte transversal se observó la asociación entre las alteraciones de la posición al amamantar, dificultades en la toma del pezón y la succión por parte del bebé con la aparición de grietas en el pezón ${ }^{(9)}$.

Se ha observado que el uso del chupete y el biberón está relacionado con el aumento del dolor en el pezón, con la aparición de grietas, congestión y reducción en la frecuencia y duración de la lactancia ${ }^{(3)}$.

En un ensayo clínico se comparó la formación materna prenatal en lactancia con la formación impartida en sesiones individuales tras el parto en 158 mujeres, observándose que el dolor en el pezón era significativamente mayor en las mujeres a las que les fueron impartidas sesiones individuales.

En un estudio clínico con 177 mujeres primíparas se evaluó el uso de compresas de agua tibia para aliviar el dolor en los pezones, observándose que en este grupo la reducción del dolor fue mayor que en el grupo control. Otro estudio comparó la aplicación de lanolina con la aplicación de compresas frías no encontrándose diferencias entre ambos tratamientos en la reducción del dolor.

Se ha encontrado que el uso de pezoneras no disminuye el dolor e incluso aumenta las lesiones por rozamiento ${ }^{(8)}$.

Se ha demostrado que la clave en la prevención del dolor y las grietas en el pezón es un buen acoplamiento boca-pezón pero se puede aplicar unas gotas de leche materna para mejorar las lesiones. No se ha demostrado que ninguna loción aplicada 
en el pezón para mejorar las lesiones sea mejor que la aplicación de leche materna, siempre y cuando se corrija el acoplamiento ${ }^{(9)}$.

\section{- Congestión mamaria}

La congestión mamaria se define como la inflamación y distensión de las mamas; suele aparecer entre el segundo y el quinto día postparto. Se relaciona con el cambio del calostro a leche más abundante y madura.

Con frecuencia suele aparecer tras el transcurso de la lactancia, las madres suelen experimentar un aumento de volumen mamario bilateral, con calor, dolor 0 endurecimiento mamario.

Se han identificado 5 variables relacionadas con la congestión mamaria:

$\checkmark$ Inicio tardío de la lactancia: se ha encontrado mayores niveles de congestión mamaria en mujeres sometidas a parto por cesárea que en mujeres sometidas a partos vaginales. Este hecho se ha relacionado con el retraso en el inicio de la lactancia de las mujeres sometidas a cesárea.

$\checkmark$ Mamadas infrecuentes e inefectivas.

$\checkmark$ Mamadas restringidas en el tiempo.

Los dos últimos parámetros están asociados a un vaciado incompleto de la mama que incrementa el riesgo de congestión mamaria. Algunas técnicas para amamantar se han asociado específicamente con una menor incidencia de congestión mamaria, entre ellas el vaciado de una mama en cada toma y alternar la mama que se ofrece primero ${ }^{(10)}$.

El amamantamiento frecuente durante las primeras 48 horas está asociado con niveles inferiores de congestión mamaria posteriores.

El manejo óptimo de la lactancia materna y el apoyo a esta se reconoce como un factor positivo en la reducción de la frecuencia de síntomas de congestión mamaria.

La buena posición, el agarre del bebé y la alimentación frecuente se ha relacionado con la incidencia de menor congestión mamaria ${ }^{(11,12) .}$.

En un estudio realizado donde se comparó a niños alimentados con limitaciones en el número o duración de las tomas con niños con alimentación a libre demanda, se observó que la lactancia con restricciones produce un aumento en el abandono de la lactancia a las 4-6 semanas posparto, un aumento de grietas en el pezón y de la turgencia mamaria y un aumento en la suplementación de la lactancia con fórmulas ${ }^{(13)}$.

El tratamiento de la congestión mamaria consiste en el vaciamiento frecuente de la mama, idealmente por el niño pero si la congestión es severa, primero debe vaciarse manualmente o con un sacaleches para que el niño pueda acoplarse y mamar efectivamente. El ultrasonido, el calor local y la aplicación de compresas frías tienen un efecto moderado en la disminución de la congestión mamaria según los estudios consultados. Sin embargo, el masaje mamario antes del amamantamiento y la toma de antiinflamatorios se ha demostrado que son eficaces en la disminución de la congestión mamaria, pero sin duda, la prevención es el mejor tratamiento ${ }^{(11,14)}$. 
- Mastitis inflamatoria e infecciosa.

La mastitis constituye la inflamación de la mama, que puede estar acompañada o no por infección. Se produce aproximadamente en un 5-33\% de las mujeres que amamantan, siendo entre un 3 y $12 \%$ bilaterales ${ }^{(15,16)}$. La clínica se caracteriza por dolor, eritema, induración y aumento del calor local en una zona de la mama, generalmente unilateral, cefalea, mialgias, compromiso del estado general y fiebre con escalofríos. Los gérmenes causales más frecuentes de la mastitis infecciosa son S. aureus, E. Coli, Streptococcus sp y Haemophilus sp.

En la mayoría de los casos la mastitis es producida por una mala posición del lactante al pecho, que lesiona el pezón e impide el vaciado adecuado de la mama, lo que origina estasis intraductal, ingurgitación e inflamación y predispone a la infección ${ }^{(17)}$.

Es importante que el niño vacíe completa y alternadamente cada uno de los pechos para evitar el acúmulo de leche que puede ocasionar el desarrollo de una mastitis ${ }^{(1,3)}$.

El tratamiento de la mastitis consta de: vaciamiento frecuente de la mama, reposo, líquidos abundantes, analgésicos o AINES y antibióticos durante 10-14 días.

En un ensayo clínico realizado se concluye que la continuidad de la lactancia en el caso de mastitis con un vaciado efectivo de la mama acorta la duración de la mastitis y disminuye la tasa de abandono precoz de la lactancia ${ }^{(18)}$.

Otro estudio dio a conocer que la evaluación del acoplamiento del bebé al pecho, el apoyo emocional, la educación y el apoyo a la lactancia materna continua son beneficiosos en la prevención y en el manejo efectivo de la mastitis ${ }^{(19)}$.

Se ha demostrado que la aplicación de frío y de masaje entre tomas alivia el dolor en la mayoría de las mujeres.

Se ha considerado beneficioso la continuidad de la lactancia en mujeres afectadas de mastitis como prevención de abscesos mamarios ${ }^{(20)}$.

En caso de mastitis recurrentes, es necesario verificar la adherencia al tratamiento y las causas predisponentes como la congestión mamaria ${ }^{(19,20)}$.

\section{DISCUSIÓN Y CONCLUSIONES}

La bibliografía consultada coincide en la asociación entre los problemas tempranos de las mamas con una técnica inadecuada, pero en algunos estudios no hay un consenso en cuanto a la metodología de investigación.

Se expone la relación de un mal acoplamiento mamario y una técnica inadecuada en la toma con la aparición de grietas en el pezón, congestión mamaria y mastitis.

También se concluye que la educación materna prenatal como método de prevención es el mejor tratamiento para estas patologías, campo donde el profesional sanitario desarrolla una labor importante. 
Por lo tanto, una técnica de amamantamiento correcto constituye la base de una lactancia exitosa y de prevención de problemas mamarios asociados a la lactancia materna como grietas en el pezón, ingurgitación mamaria o mastitis.

Por ello los profesionales de la salud deben ayudar a la prevención de dichos problemas mediante el asesoramiento tras el nacimiento, la observación de las primeras tomas y la intervención adecuada en cada caso, para evitar el abandono precoz de la lactancia.

\section{REFERENCIAS BIBLIOGRÁFÍCAS}

1. Comité de Lactancia Materna de la Asociación Española de Pediatría. Lactancia materna: guía para profesionales. Majadahonda (Madrid), Monografías de la AEP nำ; 2004.

2. Lawrence RA, Lawrence RM. Breastfeeding. A guide for the medical profession 6 th ed. Philadelphia (PA): Elsevier Mosby; 2005.

3. Hernández Aguilar MT, Aguayo Maldonado J. La Lactancia materna. Cómo promover y apoyar la lactancia materna en la práctica pediátrica. Recomendaciones del Comité de Lactancia de la AEP. An Pediatr. 2005; 63:340-356.

4. Britton C, McCormick FM, Renfrew MJ, Wade A, King SE. Apoyo para la lactancia materna. The Cochrane Plus Database. 2008; (3).

5. Parellada Esquius N, García Casanova MC, Pi Juan M, García Casanova S, Ruiz Mariscal E. Lactancia materna: ¿puede el personal sanitario influir positivamente en su duración? Aten Primaria. 2005; 35:295-300.

6. Renfrew MJ. Time to get serious about educating health professionals. Matern Child Health. 2006, 2(4):193-195.

7. Blair A, Cadwell K, Turner-Maffei C, Brimdyr R. The relationship between positioning, the breastfeeding dynamic, the lactching process and pain in breastfeeding mothers with sore nipples. Breastfeeding Rev. 2003; 11:5-10.

8. Morland-Schultz K, Hill PD. Prevention of and therapies for nipple pain: a systematic review. J Obstet Gynecol Neonatal Nurs, 2005, 34:428-437.

9. Moraes M; Da Silva L, Faliu B, Sosa C. Técnica de alimentación a pecho y aparición de trauma del pezón previo al alta hospitalaria. Arch Pediatr Urug. $2011 ; 82(1) ; 10-17$.

10. Betzold CM. An update on the recognition and management of lactational breast inflammation. J Midwifery Womens Health. 2007; 52 (6): 595-605.

11.Snowden HM, Renfrew MJ, Woolridge MW. Tratamientos para la ingurgitación mamaria durante la lactancia. The Cochrane Plus Database. 2006; (4).

12. Royal Collage of Midwives. Lactancia Maternal: Manual para profesionales.Barcelona: ACPAM ed; 1994.

13. Renfrew MJ Restricted Schedule of breastfeeding. The Cochrane Pregnancy and Childbirth Database. The Cochrane Plus Database. 1995; (2).

14. Moon JL, Humenick SS, Breast Engorgement: Contributing variables and variables amenable to nursing intervention. J Obstet Gynecol Nurs. 1989; 18 (4): 309-15.

15. Kinlay JR, O'Connell DL, Kinlay S. Incidence of mastitis in breastfeeding women during the six months after delivery: a prospective cohort study. Med $\mathrm{J}$. 1998; 169 (6):310-312.

16. Mass S. Breast Pain: engorgement, nipple pain and mastitis. Clin Obstet Gynecol. 2004; 47:676-682. 
17. World Health Organization: Mastitis: Causes and Management. Geneva: WHO/FCH/CAH/00.13. 2000.

18. Crepinsek MA, Crowe L, Michener K, Smart NA. Interventions for preventing mastitis after childbirth. Cochrane Database Syst Rev. 2010; (8):CD007239.

19. Cusack L, Brennan M. Lactational mastitis and breast abscess: diagnosis and management in general practice. Aust Fam Physician. 2011; 40 (12): 976-9.

20. Bertrand $\mathrm{H}$, Rosenblood LK. Stripping out pus in lactational mastitis: a means of preventing breast abscess. CMAJ. 1991; 145 (4): 299-306. 